Flórez, L. López, J. \& Vílchez, R.A. (2020). Niveles de resiliencia y estrategias de afrontamiento: reto de las instituciones de educación superior. Revista Electrónica Interuniversitaria de Formación del Profesorado, 23(3), 3547.

DOI: https://doi.org/10.6018/reifop.438531

\title{
Niveles de resiliencia y estrategias de afrontamiento: reto de las instituciones de educación superior
}

Lidia Flórez de Alvis, Jésica López Coronado, Rafael Alberto Vílchez Pírela

Corporación Universitaria del Caribe (CECAR)

\section{Resumen}

En este estudio se indagó sobre los niveles de resiliencia y las estrategias de afrontamiento utilizadas por los estudiantes universitarios víctimas del conflicto armado colombiano, mediante la aplicación de dos instrumentos contextualizados y validados: la Escala de Resiliencia de Wagnild y Young (1993), y la Escala Coping Modificada (EEC-M), Colombia. Los resultados revelan que el nivel de resiliencia donde se ubica el mayor número de estudiantes es el medio, con un 29.2\%; le sigue el muy alto, con un 23.3\%; el alto, con un $22.3 \%$; el bajo, con un 15.5\%; y el muy bajo, con un $9.7 \%$. Las estrategias de afrontamiento a las que más recurren los educandos son: religión, solución de problemas y reevaluación positiva; y a las que menos acuden son: espera, reacción agresiva, autonomía, búsqueda de apoyo profesional, expresión de la dificultad de afrontamiento y negación. Además, las estrategias más utilizadas en relación con los cinco niveles de resiliencia son: solución de problemas, religión, búsqueda de apoyo social y evitación emocional; y las menos usadas son: autonomía, negación, expresión de dificultad de afrontamiento y reacción agresiva.

\section{Palabras clave}

Resiliencia; niveles de resiliencia; estrategias de afrontamiento; estudiantes universitarios.

\section{Contacto:}

Lidia Flórez de Alvis, lidia.florez@cecar.edu.co, kilómetro 1, Carretera Troncal de Occidente, vía Corozal, CECAR, Sincelejo, Colombia. Artículo producto de la investigación Niveles de resiliencia y estrategias de afrontamiento: reto de la educación superior. 


\title{
Resilience levels and coping strategies: Challenge of higher education institutions
}

\begin{abstract}
This study investigated the levels of resilience and the coping strategies used by university students who are victims of the Colombian armed conflict, through the application of two contextualized and validated instruments: the Wagnild and Young Resilience Scale (1993), and the Modified Coping Scale (EEC-M), Colombia. The results reveal that the level of resilience where the highest number of students is located is the medium, with $29.2 \%$; it is followed by the very high, with $23.3 \%$; the high, with $22.3 \%$; the low, with $15.5 \%$; and the very low, with $9.7 \%$. The coping strategies to which learners resort most are: religion, problem solving and positive re-evaluation; and those to which they resort least are: waiting, aggressive reaction, autonomy, seeking professional support, expressing coping difficulty and denial. In addition, the most widely used strategies in relation to the five levels of resilience are: problem solving, religion, seeking social support and emotional avoidance; and the least used are: autonomy, denial, expressing coping difficulties and reacting aggressively.
\end{abstract}

\section{Key words}

Resilience; resilience levels; coping strategies; university students.

\section{Introducción}

La universidad, por ser el espacio donde los jóvenes construyen gran parte de su identidad, tiene el reto de constituirse como una institución educativa resiliente que engendre ilusión, alegría y optimismo en sus educandos. Así mismo, debe desarrollar en ellos la capacidad de asumir actitudes vitales positivas, en caso tal de que su horizonte de vida se les torne incierto, triste, adverso o violento como, por ejemplo, cuando están expuestos a diferentes situaciones de riesgo: desastres, vejaciones, conflicto armado, entre otras.

Es por esto que ante el panorama incierto e incluso desolador que pueden experimentar las personas en su vida cotidiana, la resiliencia debe florecer en el ámbito educativo con la esperanza de sacar del dolor una fuerza creadora que suscite en los integrantes de la comunidad académica una serie de estrategias para afrontar de manera proactiva las crisis que experimenten en su existencia.

En este sentido y teniendo en cuenta que la resiliencia es genética, pero también puede ser aprendida; este estudio tuvo como objetivo describir los recursos resilientes y las estrategias de afrontamiento empleadas por los estudiantes universitarios que han sido víctimas del conflicto armado en Colombia, determinándose primero los niveles de resiliencia, luego los recursos resilientes y las estrategias de afrontamiento, para finalmente contrastar estos comportamientos. Lo anterior es importante para desarrollar competencias no solo cognitivas, sino también sociales, emocionales y afectivas que les sirvan a los individuos para convertir la resiliencia en un hábito individual, grupal y comunitario con el cual puedan proyectar un futuro mejor y tener una vida digna. 


\section{Resiliencia y futuros exitosos posibles}

La realización de esta investigación requirió de la revisión de distintos referentes teóricos y bibliográficos que permitieron establecer un marco de referencia con el objetivo de comprender el constructo resiliencia y su aplicabilidad en el contexto educativo universitario.

Wagnild \& Young (1993) plantean que la resiliencia es la habilidad de convertir las desgracias en éxitos y se compone de dos factores: uno referido a la competencia personal y el otro, a la aceptación de uno mismo. Estos son moduladores de las dimensiones asociadas a los efectos del estrés como son la ecuanimidad, la perseverancia, la confianza en sí mismo, la satisfacción personal y el sentirse bien solo, las cuales posibilitan logros significativos de niveles de adaptabilidad y superación de infortunios. Es decir, para estos autores, la resiliencia consiste en resistir ante las dificultades, actuar de manera correcta, incluso en condiciones desventajosas, superarlas y salir transformado o fortalecido.

A su vez, Rutter (1993) considera que la resiliencia es una construcción teórica cimentada en la convicción de que algunas personas, más que otras, tienen la facultad de soportar la adversidad. De igual forma, Masten (2001) la entiende como un "fenómeno común que se produce en la mayoría de los casos del funcionamiento de los sistemas adaptativos humanos básicos" (p. 227); y Vanistendael y Lecomte (2002) la conceptúan "como un recurso que lleva a muchas personas resilientes a mostrar una extraña mezcla de fuerza y fragilidad. La fragilidad proviene de la prueba vivida, la fuerza de la prueba superada” (p. 157).

Por su parte, Cyrulnik (2001) la define "como un proceso diacrónico y sincrónico: las fuerzas biológicas de desarrollo se articulan con el contexto social para crear una representación de sí que permite la historización del sujeto" (p.40). Por ello, según García y Domínguez (2013), "es decisivo el papel de la familia, la institución educativa, la comunidad y la sociedad, como facilitadoras de la autogestión del desarrollo personal y social” (p.74) Además, Vílchez (2016) considera la estrecha relación entre la resiliencia y la dimensión espiritual, al incluir los atributos divinos entre sus elementos constitutivos.

Dentro del realismo de la esperanza, Forés y Grané (2018) expresan que la resiliencia "es una metáfora generativa que construye futuros posibles sobre la esperanza humana y la consecución de la felicidad ante los sufrimientos, los traumas y el dolor padecido" (p. 23), hasta llevarla hoy más allá de la resiliencia clásica para posicionarla como la resiliencia generativa. Esta última es definida por Grané y Forés (2019) como el arte de generar alternativas al experimentar sucesos sorpresa con el fin de asumir la incertidumbre y complejidad del mundo de la mejor manera.

La resiliencia, entonces, es muy importante para la vida, puesto que permite enfrentar las situaciones difíciles de una forma positiva; y su desarrollo depende tanto de recursos personales como externos entre los que se encuentran la familia, comunidad y las instituciones educativas. Estos medios de socialización se convierten en factores protectores que posibilitan disminuir las consecuencias negativas de las circunstancias estresantes 0 adversas. Por consiguiente, si se quiere construir resiliencia, es fundamental fortalecer las relaciones entre dichos factores, debido a que estos "ayudan al individuo a sobrellevar o generar un cambio de todos aquellos aspectos negativos y peligrosos que atenten contra su equilibrio y desarrollo" (Caldera, Aceves y Reynoso, 2016, p. 229).

En este orden de ideas, es necesario resaltar que:

Las distintas definiciones de resiliencia enfatizan en características de los sujetos, tales como habilidad, adaptabilidad, baja susceptibilidad, enfrentamiento efectivo, capacidad, competencia, resistencia a la destrucción, conductas vitales positivas, temperamento especial y habilidades cognitivas, todas desplegadas frente a 
situaciones estresantes que les permiten superarlas. También se destaca que la resiliencia se desarrolla en función de procesos sociales e intra-psíquicos; está en función de los procesos interacciónales entre las personas y estos, a su vez, aportan a la construcción del sistema intrapsíquico del ser humano (García y Domínguez, 2013, p. 66).

En consecuencia, el constructo resiliencia es multivariado y son diversas las acepciones que los autores proponen de esta variable, con la finalidad de comprender el comportamiento de las personas cuando estas enfrentan dificultades o situaciones desventajosas, haciendo uso de recursos personales o estrategias de afrontamiento que no solo les permiten superarlas, sino también trascenderlas y salir fortalecidas.

Por lo antes expuesto, la resiliencia es la competencia que invita a aprovechar y potenciar los aspectos positivos con los que cuentan los seres humanos en su individualidad, pero también en su contexto, en la búsqueda de su propio desarrollo. Asimismo, permite que se genere un bienestar biopsicosocial al superar las adversidades, lo que consecuentemente crea calidad de vida. Así, la resiliencia se convierte en una base sobre la cual los Estados pueden crear políticas educativas de prevención e intervención de resiliencia para el desarrollo humano de sus habitantes.

Con fundamento en la estrategia didáctica de la metáfora, utilizada por Forés y Grané (2018), y la del oxímoron, propuesta por Cyrulnik (2001), como figuras que posibilitan una mejor comprensión de la resiliencia, al igual que de su importancia para el crecimiento y desarrollo del ser humano al potenciar sus dimensiones biológica, psicológica, social, afectiva, emocional, religiosa y cognitiva; se define la resiliencia como el jardín sonriente que llena de alegría el corazón, pese a las adversidades. El jardín representa al ser humano que sonríe a la vida como salida inteligente de afrontamiento y aceptación de la realidad; es un reconocimiento de sí mismo y del infortunio para hacer el tránsito de la oscuridad a la luz de la esperanza, de la mano de un jardinero. Este último esta personificado en la educación por un tutor dispuesto a compartir sus sabios consejos con el fin de regar y fertilizar las ilusiones de los estudiantes, las cuales han de afianzarse con el uso de estrategias de afrontamiento requeridas para salir fortalecidos y transformados de la adversidad, lo que sin duda les debe dejar grandes aprendizajes.

\section{La resiliencia en el ámbito educativo}

La resiliencia se incorpora al ámbito educativo como un apoyo a la formación integral de los estudiantes, puesto que ellos se forman no solo adquiriendo conocimientos, sino también aprendiendo habilidades para enfrentarse a la vida. En este sentido, Werner y Smith (1989) la consideran como una estrategia pedagógica que posibilita el desarrollo de potenciales aptitudes y habilidades las cuales pueden ser modificables, adquiridas y enseñadas. Igualmente, puede convertirse en un factor social que favorece el proceso de humanización, el cual conduce a niveles superiores de autonomía, inteligencia y solidaridad.

Tal como lo plantea Melillo (2004), para los centros educativos, es un gran reto llegar a ser entornos en donde la resiliencia pase de ser una condición psicológica a una social. Para que esto ocurra, dichos centros deben estar comprometidos con la búsqueda de la justicia y el bienestar de las personas que sufren desigualdades, como lo son los estudiantes y sus familias quienes en su diario vivir sufren situaciones socioemocionales que ponen en riesgo no solo su presente y futuro, sino también los de la sociedad en general.

A lo anterior se suma que las problemáticas con las que están llegando los estudiantes al aula, como por ejemplo, las desventajas socioeconómicas, la violencia intrafamiliar y social, el uso inadecuado de los medios tecnológicos, el consumo de sustancias psicoactivas, el manejo 
irresponsable de su sexualidad, la participación directa o indirecta en los conflictos armados, entre otras; están ocasionando en ellos intolerancia, desinterés por el estudio y poca preocupación por su futuro.

Por lo tanto, "recordar a los educandos lo importantes que son para la sociedad, resulta ser una de las estrategias más útiles para promover la resiliencia en los contextos escolares" (López, 2010, p. 11). Esta premisa cobra relevancia en la educación superior, pues las universidades deben convertirse en centros resilientes que les permitan a sus discentes desarrollar todo su potencial cognitivo, personal y social para que tanto ellos como la sociedad se vean beneficiados. Es por esto que se debe:

Recuperar el acercamiento positivo a la resiliencia como momento de potenciación y transformación personal en la educación superior, a pesar de las inminentes adversidades del intenso crecimiento personal; construyendo, de esta forma, valiosos recursos de empoderamiento para el educando en un mundo altamente cambiante. (Belykh, 2018, pp. 278-279).

Además, cuando los alumnos desarrollan resiliencia, muestran mayor motivación hacia su aprendizaje y obtienen mejores resultados académicos, debido a que "los adolescentes resilientes se sienten más identificados con la escuela, invierten más en la tarea de aprendizaje y también, aunque en menor medida, son más participativos que los no resilientes" (Rodríguez, Ramos, Ros, Fernández y Revuelta, 2016, p. 81). En esta construcción de entornos académicos resilientes, el docente desempeña un papel fundamental, puesto que:

Pasa de ser un tutor académico a ser un tutor de resiliencia, ya que no es posible afrontar la adversidad y la incertidumbre si no se establecen relaciones afectivas seguras. Nuestro alumnado nos está reclamando con insistencia la figura de un Educador que se vincula con ellos para no solo apoyarles de forma incondicional cuando tienen problemas, sino también para que los animen a arriesgarse, a expandirse y a no conformarse. (Forés y Grané, 2018, p. 127).

Por lo antes expuesto, la educación hoy debe implementar pedagogías emergentes que formen a las personas para enfrentar con actitud positiva un mundo volátil donde nada es sólido, todo puede suceder y nada puede ocurrir con certeza absoluta. Es decir, un mundo configurado por acontecimientos sorpresa a los cuales hace alusión Taleb (2007) con el nombre de cisnes negros.

\section{Estrategias de afrontamiento: el saber enfocarse}

El concepto de estrategia de afrontamiento ha sido trabajado en el campo de la salud mental y la psicopatología principalmente, relacionándolo con el estrés, las emociones y la solución de problemas (Londoño et al., 2006). Entre los principales investigadores de esta temática se encuentran Chorot y Sandín (1993), quienes elaboraron la Escala de Estrategias de Coping (EEC-R), con el objetivo de lograr una prueba psicométrica que evaluara las diferentes formas en que las personas enfrentan situaciones estresantes. Esta escala fue adaptada al contexto colombiano por los investigadores Londoño et al. (2006), quienes la denominaron Escala de Estrategias de Coping - Modificada (EEC-M), la cual presenta 12 factores que evidencian la manera en que las personas mueven sus recursos cognitivos y comportamentales para superar la adversidad. Estos factores son: solución de problemas, búsqueda de apoyo social, espera, religión, evitación emocional, búsqueda de apoyo profesional, reacción agresiva, evitación cognitiva, reevaluación positiva, expresión de la dificultad de afrontamiento, negación y autonomía. A continuación, se resume cada uno de ellos. 
Con la solución de problemas, se realizan múltiples acciones cognitivas y analíticas para examinar las situaciones problemáticas, intervenirlas y resolverlas. Con la búsqueda del apoyo social, se espera la ayuda emocional de los seres humanos más cercanos, como lo son los integrantes de la familia, los amigos u otros para tolerar o enfrentar las dificultades. Con la espera, se cree que la situación problema se resolverá de manera positiva por sí sola al transcurrir el tiempo. Con la religión, se desarrolla una relación paternal con un ser superior llamado Dios, por consiguiente, el rezo y la oración contribuyen de manera positiva para sobrellevar el problema o para buscarle una solución. Con la evitación emocional, se movilizan recursos con el fin de esconder y reprimir las propias emociones, es decir, los individuos evitan dar a conocer el problema, por las reacciones emocionales negativas que esto les genera o por creer que serán desaprobados socialmente. Con el apoyo profesional, que a su vez se constituye en un apoyo social, se recurre a especialistas con el propósito de recibir información sobre un problema y conocer las posibles formas como puede enfrentarse, lo cual aumenta el control percibido.

Con la reacción agresiva, se disminuye la carga emocional en un momento determinado, cuando los sujetos expresan la impulsividad de la ira hacia sí mismos, los objetos o las demás personas. Con la evitación cognitiva, se tiende a desarrollar múltiples actividades con el objetivo de neutralizar los pensamientos negativos y perturbadores. Con la reevaluación positiva, se le cambia el significado a la situación, puesto que se forjan pensamientos optimistas al identificar los aspectos positivos del problema y al considerar que la solución de las dificultades genera unos aprendizajes para toda la vida.

Con la expresión de la dificultad de afrontamiento, se enfrentan las emociones negativas producidas por la situación o se identifica el problema y se buscan soluciones por medio de recursos que no son propios. Con la negación, se sobrelleva o soporta el problema como si no existiera, tratando de no pensar en él o alejándose de todo lo que se relaciona con este. Por último, con la autonomía, se busca solucionar el problema de forma independiente, sin contar con el apoyo de otras personas tales como amigos, familiares o profesionales.

Por lo tanto, el afrontamiento se puede dirigir al problema o a la emoción. En el primer caso, la persona busca solucionar la dificultad con el objetivo de volver al estado de bienestar y tranquilidad que le fue quitado al enfrentar la crisis. En el segundo caso, la persona busca autorregularse, de tal manera que se disminuya el efecto negativo que el problema ha producido en sus emociones. Lo anterior implica que los estilos de afrontamiento dependen de las características personales de cada individuo.

\section{Metodología}

\section{Diseño de Investigación}

Esta investigación se centró en describir los niveles de resiliencia de los estudiantes universitarios víctimas del conflicto armado colombiano e identificar las estrategias de afrontamiento utilizadas por los mismos. Está enmarcada en el paradigma positivista y basada en una metodología cuantitativa. Asimismo, es un estudio no experimental de tipo transversal y descriptivo.

\section{Participantes}

Los participantes de este estudio son 103 estudiantes universitarios que han sido víctimas del conflicto armado en Colombia. Estos pertenecen a siete Instituciones de educación superior del departamento de Sucre y son beneficiarios de las becas que se les han ofrecido a las víctimas de este conflicto en el país, específicamente en Sucre. 


\section{Procedimiento}

Para recolectar la información, primero se solicitó la autorización de las instituciones educativas; luego, se contactaron a los estudiantes, se les explicaron los objetivos y el alcance de la investigación, y se les pidió que firmaran voluntariamente el consentimiento informado y diligenciaran los instrumentos. Posteriormente, se inició el proceso de organización de la base de datos y análisis de la información.

\section{Instrumentos}

Para lograr los objetivos del presente estudio, se aplicaron dos instrumentos contextualizados y validados. Uno de ellos fue la Escala de Resiliencia de Wagnild y Young (1993), adaptada en Perú por Novella (2003) y constituida por 25 ítems que miden el grado de resiliencia individual, la cual es considerada como una característica de personalidad positiva que permite la adaptación del individuo a situaciones adversas. Los ítems están distribuidos en dos grandes factores: competencia personal y aceptación de uno mismo. A su vez, estos están integrados por cinco dimensiones: ecuanimidad, perseverancia, confianza en sí mismo, satisfacción personal y sentirse bien solo. Esta escala es fiable, pues presentó un Alfa de Cronbach de 0.853 , y permitió describir los niveles de resiliencia de los universitarios que han sido víctimas del conflicto armado colombiano. También se aplicó la Escala Coping Modificada (EEC-M) - Colombia, con el fin de identificar las estrategias de afrontamiento empleadas por los participantes mencionados anteriormente. Esta escala está conformada por 12 factores que se resumieron en el apartado anterior y su Alfa de Cronbach fue de 0.842 , lo cual significa que el instrumento tiene una alta confiabilidad.

\section{Análisis de datos}

El procesamiento y análisis de los datos se realizó ejecutando el programa estadístico SPSS, versión 25. Básicamente, se realizaron análisis descriptivos de la información obtenida en la aplicación de los instrumentos, la cual se organizó en frecuencias absolutas, porcentajes y análisis correlacional para establecer la asociación entre aspectos fundamentales para las escalas utilizadas. Para las pruebas de consistencia interna, se usó el Alfa de Cronbach.

\section{Resultados}

\section{Niveles de Resiliencia}

Tabla 1.

Niveles de Resiliencia

\begin{tabular}{ccccccc}
\hline \multirow{2}{*}{$\begin{array}{c}\text { Niveles de } \\
\text { Resiliencia }\end{array}$} & \multicolumn{2}{c}{ General } & \multicolumn{2}{c}{$\begin{array}{c}\text { Factor I. } \\
\text { Competencia personal }\end{array}$} & \multicolumn{2}{c}{$\begin{array}{c}\text { Factor II. } \\
\text { Aceptación de uno mismo }\end{array}$} \\
\cline { 2 - 7 } & Frecuencia & Porcentaje & Frecuencia & Porcentaje & Frecuencia & Porcentaje \\
\hline Muy Bajo & 10 & 9.7 & 10 & 9.7 & 10 & 9.7 \\
Bajo & 16 & 15.5 & 16 & 15.5 & 19 & 18.4 \\
Medio & 30 & 29.2 & 29 & 28.2 & 24 & 23.3 \\
Alto & 23 & 22.3 & 24 & 23.3 & 24 & 23.3 \\
Muy Alto & 24 & 23.3 & 24 & 23.3 & 26 & 25.3 \\
\hline
\end{tabular}


De manera general, en la tabla 1 se registra que los niveles de resiliencia de la población objeto de estudio, son los siguientes: el $45.6 \%$ de los sujetos de estudio se ubican en los niveles superiores de la escala (muy alto y alto); el $29,2 \%$ en un nivel intermedio y el $25,2 \%$ en los niveles inferiores (bajo y muy bajo). Es de resaltar que en el factor 1 (competencia personal) y en el factor 2 (aceptación de uno mismo), los porcentajes de estudiantes que se ubicaron en los niveles superiores y el intermedio fueron del $74.8 \%$ y $71.9 \%$, respectivamente.

Estrategias de afrontamiento

Tabla 2.

Estrategias de Afrontamiento según Escala Coping Modificada (EEC-M)

\begin{tabular}{|c|c|c|c|c|c|c|}
\hline Estrategias de afrontamiento & $\mathbf{N}$ & $\mathbf{C N}$ & $\mathbf{A V}$ & $\mathbf{F R}$ & $\mathbf{C S}$ & $\mathbf{S}$ \\
\hline Solución de Problemas & 1.5 & 5.8 & 18.6 & 19.4 & $\mathbf{3 5 . 5}$ & $\mathbf{1 9 . 2}$ \\
\hline Búsqueda de Apoyo Social & 8.7 & 14.7 & 25.5 & 17.5 & 20.5 & 13.0 \\
\hline Espera & $\mathbf{3 5 . 8}$ & $\mathbf{3 1 . 6}$ & 20.9 & 5.0 & 5.4 & 1.3 \\
\hline Religión & 8.3 & 7.8 & 17.5 & 12.2 & $\mathbf{1 4 . 6}$ & $\mathbf{4 0 . 1}$ \\
\hline Evitación Emocional & 10.1 & 18.6 & 30.7 & 17.5 & 16.4 & 9.7 \\
\hline Búsqueda de Apoyo Profesional & $\mathbf{2 3 . 1}$ & $\mathbf{2 8 . 3}$ & 22.7 & 10.1 & 11.1 & 4.7 \\
\hline Reacción Agresiva & $\mathbf{3 5 . 7}$ & $\mathbf{3 0 . 9}$ & 21.9 & 5.6 & 4.5 & 1.4 \\
\hline Evitación Cognitiva & $\mathbf{7 . 4}$ & 16.3 & 35.4 & 14.0 & 17.7 & 9.3 \\
\hline Reevaluación Positiva & 2.4 & 6.6 & 21.7 & $\mathbf{2 0 . 2}$ & $\mathbf{2 9 . 3}$ & $\mathbf{1 9 . 8}$ \\
\hline Expresión de la Dificultad de & $\mathbf{2 6 . 7}$ & $\mathbf{2 1 . 8}$ & 30.8 & 8.0 & 9.7 & 2.9 \\
\hline Afrontamiento & $\mathbf{1 7 . 5}$ & $\mathbf{2 5 . 6}$ & 30.7 & 13.3 & 9.4 & 3.6 \\
\hline Negación & $\mathbf{3 3 . 5}$ & $\mathbf{1 9 . 9}$ & 23.8 & 10.7 & 6.8 & 5.4 \\
\hline Autonomía & & &
\end{tabular}

N: Nunca CN: Casi Nunca AV: A Veces FR: Frecuentemente CS: Casi Siempre S: Siempre.

La tabla 2 refleja tanto las estrategias de afrontamiento más usadas como las menos usadas por la población objeto de estudio. En este sentido, las estrategias religión (54.7\%), solución de problemas $(54.7 \%)$ y reevaluación positiva (49.1\%) son a las que siempre o casi siempre recurren los estudiantes; mientras que las estrategias de espera (67.4\%), reacción agresiva (66.6\%), autonomía (53.4\%), búsqueda de apoyo profesional (51.4\%), expresión de la dificultad de afrontamiento (48.5\%) y negación (43.1\%) son las estrategias a las que nunca o casi nunca recurren.

Teniendo claro cuáles son las estrategias de afrontamiento que siempre y casi siempre utilizan los estudiantes (detalladas en el párrafo anterior), se hace necesario revisar los resultados de las acciones que porta cada uno de sus ítems, porque estos connotan la información necesaria para una atención más personalizada y oportuna. Es así como en estas estrategias se identificaron los siguientes comportamientos: en la estrategia religión, el 70.9\% tiene fe en que Dios remedie la situación, el $67.0 \%$ reza, el $54.4 \%$ deja todo en manos de Dios y el $62.1 \%$ busca tranquilizarse a través de la oración. En la estrategia solución de problemas, el $65.0 \%$ manifiesta que, frente a un problema, espera conocer bien la situación antes de actuar, el $60.2 \%$ analiza lo positivo y negativo de las diferentes alternativas, el $58.3 \%$ hace frente al problema poniendo en marcha varias soluciones, el $57.3 \%$ piensa que hay un momento oportuno para analizar la situación y el $55.4 \%$ no puede dar la mejor solución a los problemas hasta que no 
tenga claridad frente a la situación. En la estrategia reevaluación positiva, los sujetos de estudio se identifican con las siguientes afirmaciones: a pesar de la magnitud de la situación tiendo a sacar algo positivo (58.3\%), intento sacar algo positivo del problema (57.3\%) y trato de identificar las ventajas del problema (49.5\%).

En relación con las estrategias de afrontamiento que nunca o casi nunca utilizan, se identificaron las siguientes acciones: en la estrategia espera, el $80.5 \%$ espera a que la solución Ilegue sola, el $80.5 \%$ piensa que con el paso del tiempo el problema se soluciona, el $78.6 \%$ no hace nada porque el tiempo todo lo dice, el $76.7 \%$ se resigna y deja que las cosas pasen y el $71.9 \%$ considera que las cosas por sí solas se solucionan. En la estrategia reacción agresiva, el $72.8 \%$ expresa su rabia sin calcular las consecuencias, el 67.9\% descarga su mal humor con los demás, el $67.0 \%$ se deja llevar por su mal humor, el $64.1 \%$ se comporta de forma hostil con los demás y el $61.0 \%$ se sale de casillas. En la estrategia autonomía, el $69.0 \%$ piensa que no necesita la ayuda de nadie y menos de un profesional. En la búsqueda de apoyo profesional, el $60.2 \%$ pide ayuda a algún médico o psicólogo para aliviar su tensión, el 52.4\% procura conocer mejor el problema con la ayuda de un profesional y el $49.5 \%$ intenta conseguir más información sobre el problema acudiendo a profesionales. En la estrategia expresión de la dificultad de afrontamiento, el $67.9 \%$ no es capaz de llorar, así lo quiera y al $59.3 \%$ le es difícil pensar en posibles soluciones a sus problemas.

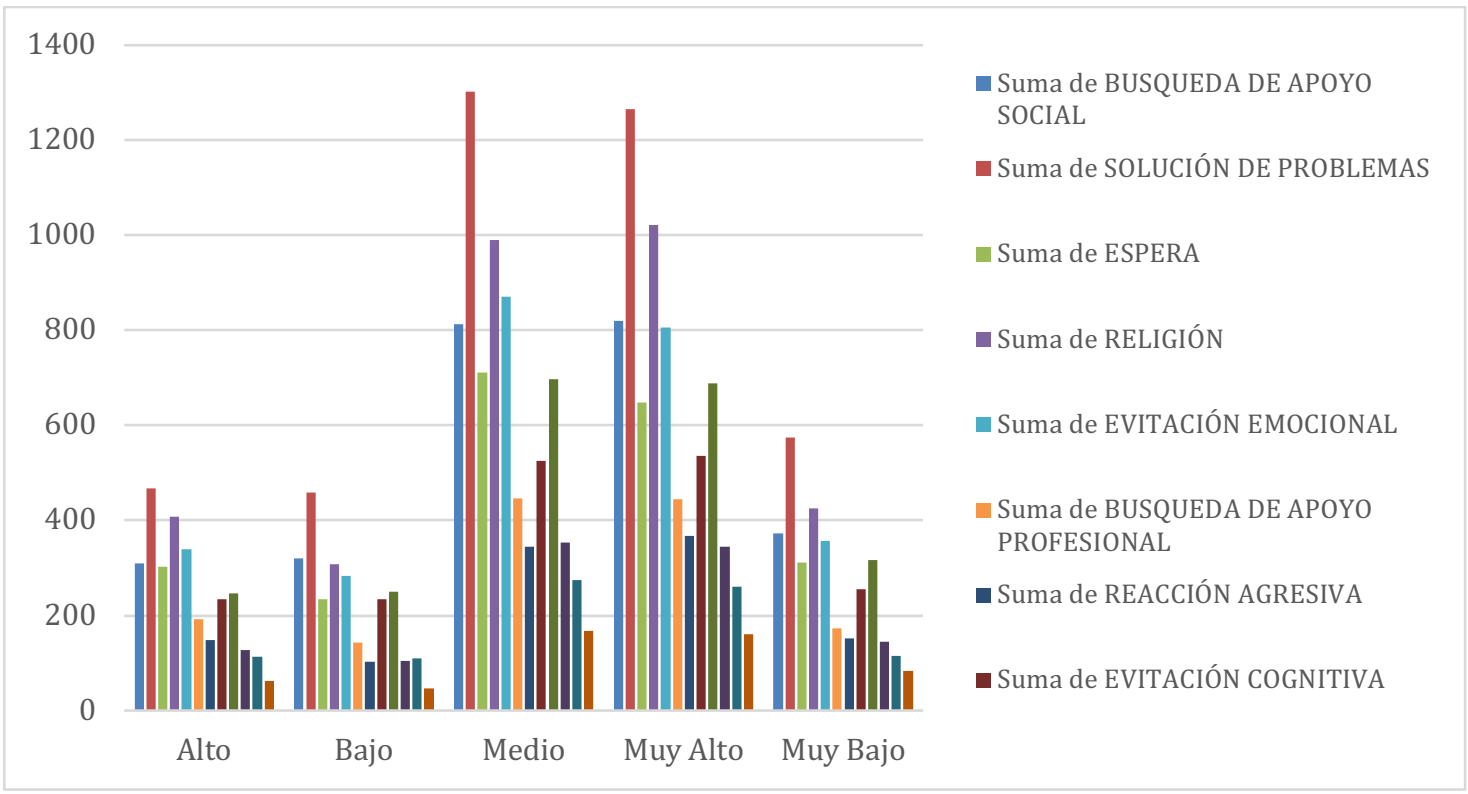

Figura 1.

Estrategias de afrontamiento vs niveles de resiliencia en estudiantes universitarios víctimas del conflicto armado en Colombia.

En la figura 1 se puede observar que los estudiantes emplean las estrategias de afrontamiento en los cinco niveles de resiliencia, pero con distinta frecuencia. Asimismo, es posible apreciar cuáles de esas estrategias son las más y menos utilizadas por los estudiantes, en general, en todos los niveles.

Entre las primeras, se encuentran en orden de importancia las siguientes: solución de problemas, religión, búsqueda de apoyo social, evitación emocional y reevaluación positiva. La espera también es una de las estrategias más usadas en la mayoría de los niveles; excepto en el nivel bajo, en el que esta es reemplazada por la evitación cognitiva. 
Entre las segundas, se ubican desde el puesto más bajo al más alto, las siguientes: autonomía, negación, expresión de la dificultad de afrontamiento, reacción agresiva y búsqueda de apoyo profesional. De igual forma, la evitación cognitiva es una de las estrategias menos utilizadas en la mayoría de los niveles; excepto en el nivel bajo, en el que esta es sustituida por la espera.

\section{Discusión y conclusiones}

En referencia al nivel de resiliencia, existe un contraste significativo entre los resultados del presente estudio, en el que los jóvenes universitarios poseen un nivel de resiliencia muy alto, y los del realizado por González, López y Valdez (2015) cuyos datos descriptivos, indican que los niños y adolescentes tienen una resiliencia baja, mientras que los adultos jóvenes y adultos medios se encuentran en el nivel de resiliencia alta. No obstante, hay coincidencias con la investigación realizada por Moreno, Fajardo, González, Coronado y Ricaurte (2019), en la que más del $80 \%$ de los niños y adolescentes poseen un nivel alto de resiliencia. En ambos casos, la población objeto de estudio utiliza los factores protectores y recursos internos para afrontar dificultades y fortalecer sus proyectos de vida.

En este mismo sentido, la investigación realizada por Quisbert (2014) muestra que de los sujetos a quienes se les aplicó el instrumento, el $29 \%$ presenta un nivel general de resiliencia alto; el 38.7\%, un nivel medio y el 32.3\%, un nivel bajo. Según el estudio, la mayoría de los niños y las niñas que pertenecen a una familia monoparental exhiben un nivel de resiliencia general medio. Además, ningún niño o niña carece de la capacidad resiliente; sin embargo, esta necesita seguir siendo fortalecida. Lo descrito concuerda con los hallazgos del presente estudio, en el cual se evidencia la capacidad resiliente en la totalidad de los jóvenes, sin descartar que en un grupo de aproximadamente $10 \%$ es necesario fortalecer factores resilientes.

Adicionalmente, Monterrosa, Ulloque y Arteta (2018) encontraron factores resilientes en jóvenes embarazadas que facilitan el éxito y la superación de adversidades, como el apoyo social, la religiosidad y espiritualidad que se asemejan y coinciden con los factores de los jóvenes universitarios de esta investigación. De igual forma, Morán et al. (2019) llevaron a cabo una investigación cuyo objetivo fue averiguar qué estrategias de afrontamiento predicen mayor resiliencia. Estos autores hallaron que la aceptación del problema, el humor y la religión tuvieron los puntajes más altos. Esto guarda correspondencia con los resultados obtenidos en los estudiantes universitarios víctimas del conflicto armado colombiano, cuyas estrategias de afrontamiento predominantes son: religión, solución de problemas y reevaluación positiva.

Asimismo, en un estudio hecho por Viñas, González, García, Malo y Casas (2015); ellos concluyen que las estrategias de afrontamiento están estrechamente ligadas al bienestar personal y resaltan entre estas el optimismo y la resolución de problemas. Por su parte, Fernández, Contini, Ongarato, Saavedra y De la Iglesia (2009), al analizar las estrategias que emplean los estudiantes de nivel medio y universitario para afrontar los problemas académicos, observaron que en estos predominan el análisis lógico, la resolución de problemas y la reevaluación positiva. Es notorio, entonces, que los resultados de ambos estudios coinciden con los de esta investigación, dado que la reevaluación positiva y la solución de problemas son estrategias que los estudiantes víctimas del conflicto siempre emplean para hacer frente a los desafíos que se les presentan.

Por el contrario, los resultados anteriores difieren de los encontrados en el trabajo de Casari, Anglada y Daher (2014), en el cual se analizan las estrategias de afrontamiento que usan los 
universitarios ante los exámenes, puesto que se encuentra que ellos recurren principalmente a la evitación cognitiva, resignación y descarga emocional; y en menor medida a la reevaluación positiva y solución de problemas al momento de superar dificultades.

Teniendo en cuenta los resultados anteriores se corrobora que la resiliencia es un constructo multidimensional que los seres humanos utilizan para salir fortalecidos de las dificultades que los arremeten y que les permite incluso alcanzar altos niveles de éxito en los proyectos que emprendan. Es así como los estudiantes víctimas del conflicto armado colombiano, expuestos a situaciones de riesgo, conservan su funcionalidad en las esferas conductual y afectiva; y a esto se le suman las excelentes competencias y habilidades sociales como atributos resilientes de su personalidad. Es importante mencionar que los jóvenes en situación de vulnerabilidad y que tienen recuerdos de hechos victimizantes, reflejan emociones negativas; pero, a pesar de ello, se muestran con mayores niveles de resiliencia ante esas circunstancias. También cabe anotar que el aprendizaje obtenido por los educandos, al aplicar estrategias con el fin de superar situaciones desventajosas, los condiciona para hacer frente a riesgos de mayor envergadura en el futuro.

En este sentido, la adaptación positiva asumida por los jóvenes universitarios ante las adversidades y los hechos victimizantes que han marcado sus vidas, promueve el desarrollo de competencias que los prepara para asumir riesgos superiores, fortalecer su inteligencia emocional y sus motivaciones intrínsecas. Es por ello, que los resultados de la investigación confirman que la resiliencia está estrechamente ligada a factores protectores que amortiguan los impactos emocionales, entre los que se pueden mencionar: el humor, la perseverancia, confianza en sí mismo, empatía y orientación a la resolución de problemas.

Lo anterior indica que la resiliencia beneficia el desarrollo de habilidades en los individuos y los hace más competentes socialmente, y capaces para resolver problemas y orientar a los sujetos hacia un mejor futuro. Este desarrollo de competencias es muy personal y suigéneris en cada individuo y no se puede generalizar partiendo de las experiencias de otras personas; lo que hace sumamente difícil su replicabilidad en diferentes contextos personales, familiares y sociales. No obstante, el uso frecuente de estrategias de afrontamiento por parte de los sujetos, ante las dificultades diarias, permite forjar su personalidad y carácter. A su vez, esto fortalece la percepción de sí mismos, su autoconfianza y sentido de vida; factores estos que sí pueden ser promovidos en diferentes escenarios.

Se concluye, entonces, que la resiliencia le abre las puertas al ser humano hacia la esperanza y le brinda la posibilidad de estar abierto a un aprendizaje continuo para transformar su vida y la de otros, a pesar de las condiciones más extremas que pueda experimentar en su entorno. Por ello, enfatiza en el potencial humano desde la responsabilidad individual y colectiva; en este sentido, a las instituciones de educación superior les asiste el compromiso social y moral de promoverla entre los miembros de su comunidad educativa, para fortalecer su uso en la resolución de problemas y toma de decisiones, que los lleven a incrementar su capacidad de defensa, así como a construir y reconstruir su vida frente a situaciones, ya sean positivas o adversas.

\section{Referencias}

Belykh, A. (2018). Resiliencia e inteligencia emocional: conceptos complementarios para empoderar al estudiante. Revista Latinoamericana de Estudios Educativos, 48(1), 255282. Recuperado de https://search-proquestcom.ezproxy.cecar.edu.co:2443/docview/2203083773?accountid=34487 
Caldera, J. F., Aceves, B. I. y Reynoso, O. U. (2016). Resiliencia en estudiantes universitarios. Un estudio comparado entre carreras. Psicogente, 19 (36) 2016, doi:http://dx.doi.org.ezproxy.cecar.edu.co:8080/10.17081/psico.19.36.1294.

Casari, L., Anglada, J. y Daher, C. (2014). Estrategias de afrontamiento y ansiedad ante exámenes en estudiantes universitarios. Revista de Psicología, 32 (2), 243-269. Recuperado de https://www.redalyc.org/articulo.oa?id=3378/337832618003

Chorot, P. y Sandín, B. (1993). Escala de Estrategias de Coping Revisado (EEC-R). Madrid: UNED.

Cyrulnik, B. (2001). La maravilla del dolor: el sentido de la resiliencia. Barcelona: Editorial Granica.

Fernández, M., Contini, N., Ongarato, P., Saavedra, E. y De La Iglesia, G. (2009). Estrategias de afrontamiento frente a problemas académicos en estudiantes medios y universitarios. Revista Iberoamericana de Diagnóstico y Evaluación - e Avaliação $\begin{array}{lllll}\text { Psicológica, } & 1 & \text { (27), 63-84. } & \text { Recuperado de }\end{array}$ https://www.redalyc.org/pdf/4596/459645443005.pdf

Forés, A y Grané, J. (2018). La resiliencia, crecer desde la adversidad. Barcelona: Plataforma Editorial.

García, C. y Domínguez, E. (2013). Desarrollo teórico de la resiliencia y su aplicación en situaciones adversas: una revisión analítica. Revista Latinoamericana de Ciencias Sociales, Niñez y Juventud, 11 (1), pp. 63-77.DOI:10.11600/1692715x.1113300812.

González, N., López, A. y Valdéz, J. (2015). Resiliencia: diferencias por edad en hombres y mujeres mexicanas. Acta de Investigación Psicológica - Registros de investigación psicológica, $\quad 5 \quad$ (2), 1996-2011. Recuperado de http://www.scielo.org.mx/pdf/aip/v3n1/v3n1a4.pdf

Grané, J. y Forés, A. (2019). Los patitos feos y los cisnes negros. Resiliencia y neurociencia. Barcelona: Plataforma Editorial.

Londoño, N., Henao, G., Puerta, I., Posada, S., Arango, D. y Aguirre. D. (2006). Propiedades psicométricas y validación de la escala de estrategias de afrontamiento modificadas (EEC-M) en una muestra colombiana. Universitas Psychologica, 5 (2), 327-349. Recuperado de https://www.redalyc.org/articulo.oa?id=647/64750210

López, T. V. (2010). Educación y Resiliencia: alas de la transformación Social. Actualidades Investigativas en Educación, 10(2), 1-14. Recuperado de https://revistas.ucr.ac.cr/index.php/aie/article/view/10130/17982

Masten, A. S. (2001). Ordinary magic: Resilience processes in development. American Psychologist, 56(3), 227-238. https://doi.org/10.1037/0003-066X.56.3.227

Melillo, A. (2004). Sobre la necesidad de especificar un nuevo pilar de resiliencia. En A. Melillo, E.B. Suárez y D. Rodríguez (Comps.), Resiliencia y subjetividad. Buenos Aires: Paidós.

Monterrosa, Á., Ulloque, L. y Arteta, C. (2018). Estrategias para mejorar el nivel de resiliencia en adolescentes embarazadas. Investigación en Enfermería: Imagen y Desarrollo, 20(2). Recuperado de https://www.redalyc.org/articulo.oa?id=145256681007

Morán, M. C., Finez, M, J., Menezes, E., Pérez, M. C., Urchaga, J. D. y Vallejo, G. (2019). Estrategias de afrontamiento que predicen mayor resiliencia. Revista Internacional de Psicología del Desarrollo y la Educación, 4 (1), 183-190. Recuperado de https://www.redalyc.org/jatsRepo/3498/349861666018/html/index.html 
Moreno, N., Fajardo, Á, P., González, A. C., Coronado, A. E. y Ricarurte, J. A. (2019). Una mirada desde la resiliencia en adolescentes en contextos de conflicto armado. Revista de investigación Psicológica, (21), 57-72. Recuperado de http://www.scielo.org.bo/pdf/rip/n21/n21_a05.pdf

Quisbert, M. M. (2014). Capacidad resiliente en niños y niñas de 9 a 12 años que pertenecen a familias monoparentales de la unidad educativa "General Esteban Arce". Revista de Investigacion Psicológica, (11), 43-66. Recuperado de http://www.scielo.org.bo/scielo.php?script=sci_arttext\&pid=S222330322014000100004\&lng=es\&tlng=es

Rodríguez, F., Ramos, D., Ros, I., Fernández, Z. y Revuelta, L. (2016). Resiliencia e implicación escolar en función del sexo y del nivel educativo en educación secundaria. Aula abierta, 44, 77-82. https://doi.org/10.1016/j.aula.2015.09.001.

Rutter, M. (1993). Resilience: Some conceptual considerations. Journal of Adolescent Health, 14 (8), 626-631. https://doi.org/10.1016/1054-139X(93)90196-V

Taleb, N. (2007). El cisne negro. El impacto de lo altamente improbable. España: Editorial planeta.

Vanistendael, S. y Lecomte, J. (2002). La felicidad es posible. Barcelona: Gedisa.

Vílchez, R. (2016). Resiliencia Espiritual: transformando la indefensión. Venezuela: UNERMB.

Viñas, F., González, M., García, Y., Malo, S. y Casas, F. (2015). Los estilos y estrategias de afrontamiento y su relación con el bienestar personal en una muestra de adolescentes. Anales de Psicología, 31 (1), 226-233. Recuperado de http://scielo.isciii.es/pdf/ap/v31n1/psico_adolescencia2.pdf

Wagnild, G. M., \& Young, H. M. (1993). Development and psychometric evaluation of the resilience scale. Journal of Nursing Measurement, 1, 165-178. ile:///C:/Users/reyde/Downloads/1054-wagnild_1993_resilience_scale_2\%20(2).pdf

Werner, E. (1989). High-risk children in young adulthood: a longitudinal study from birth to 32 years. American Journal of Orthopsychiatry, 59(1), 72-81. https://doi.org/10.1111/j.1939-0025.1989.tbo1636.x 